\title{
Specific Nucleotide Changes in the Subgenomic Promoter Region Influence Infectivity of the Sindbis Virus
}

\author{
Wu-yang Zhu ${ }^{a}$ Ting Zhao ${ }^{b}$ Guo-dong Liang ${ }^{a}$ \\ a State Key Laboratory for Infectious Disease Prevention and Control, National Institute for Viral Disease Control

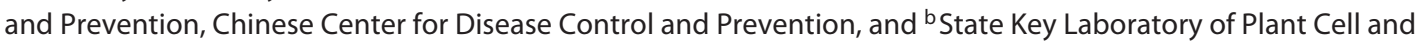 \\ Chromosome Engineering, Institute of Genetics and Developmental Biology, Chinese Academy of Sciences, \\ Beijing, China
}

\section{Key Words}

Sindbis virus · Subgenomic promoter · Infectivity

\begin{abstract}
Transcription of the subgenomic mRNA of Sindbis virus (SINV) is initiated at a subgenomic promoter (SP). Alignment of SINV sequences identified a 68-nucleotide conserved domain spanning -19 to +49 relative to the subgenomic mRNA start site. Nucleotide $T$ or $C$ is present at -18 or +49 in all known SINVs while a Sindbis-like virus XJ-160 has an A or T at a corresponding position. Our results indicate that deletion or substitution of the T at +49 decreased the activity of $\mathrm{SP}$, while substituting $T$ for $A$ at -18 did not decrease the activity of SP or genetic stability of recombinant SINV.
\end{abstract}

Copyright $\odot 2013$ S. Karger AG, Basel

Sindbis virus (SINV) is the prototype virus of the $A l$ phavirus genus and member of the Togaviridae family, which contains several significant human pathogens and potential bioterrorism or biowarfare agents, including Venezuelan, Eastern, and Western equine encephalitis virus (VEEV, EEEV, and WEEV, respectively) $[1,2]$. Members of the genus Alphavirus are similar in molecular ar- chitecture and in the pattern of events comprising viral replication; thus, less pathogenic SINVs provide an ideal system for studying the replication strategy and pathogenetic mechanism of alphaviruses. The genome of SINV consists of an approximately $11.7-\mathrm{kb}$ single-stranded RNA molecule of positive polarity, which is capped at its $5^{\prime}$ end and polyadenylated (polyA) at its $3^{\prime}$ end [3]. Early during infection, the polymerase complex uses the plussense genomic RNA to synthesize a full-length minus strand complement. The minus strand in turn is used as the template for the synthesis of daughter full-length plusstrand (49S) mRNA. In addition, the minus strand is used as template for the transcription of a smaller or subgenomic (26S) mRNA. Transcription of the 26S mRNA initiates at an internal site, known as the subgenomic promoter (SP) $[4,5]$. This subgenomic mRNA is identical to the $3^{\prime}$ third of the genomic RNA that encodes the virus' structural proteins [3], which are required for progeny virion production.

A comparison of known Alphavirus genomic sequences identified a conserved 21-nucleotide domain encompassing 19 nucleotides upstream and two downstream $(-19$ to +2$)$ relative to the $26 \mathrm{~S}$ mRNA start site [6]. This region codes for the carboxyl terminus of nsP4 on the plus strand and contains the initiation site for

\section{KARGER}

E-Mail karger@karger.com

www.karger.com/int
(C) 2013 S. Karger AG, Basel

0300-5526/13/0565-0337\$38.00/0 


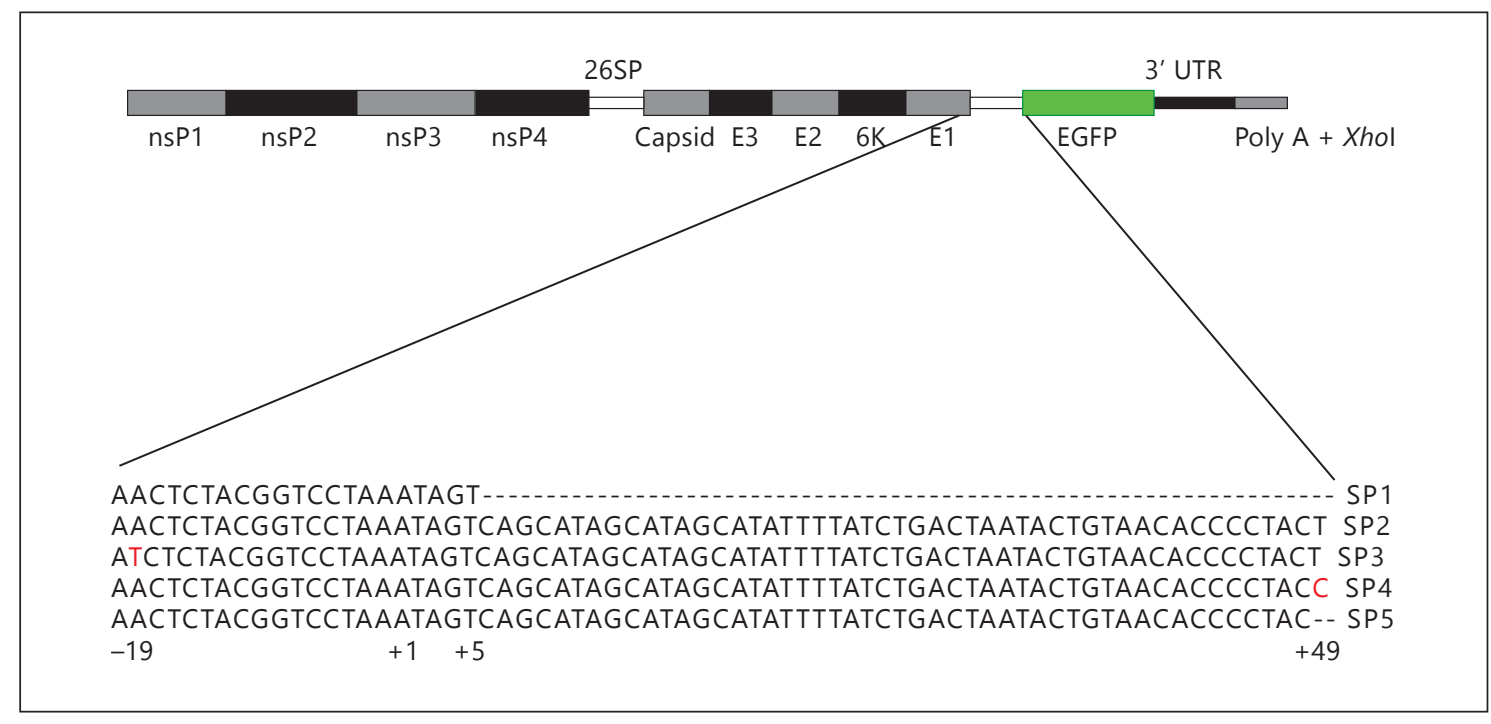

Fig. 1. Structures of derivatives containing two SPs. The designed SP was inserted immediately after the El gene of XJ-160 virus, and was followed by the reporter gene and $3^{\prime}$ UTR.

subgenomic mRNA synthesis on the minus strand. The junction region spanning -19 to +5 relative to the $26 \mathrm{~S}$ mRNA start site was identified as the minimal subgenomic mRNA promoter of SINV, which is the shortest sequence that is still active as a promoter [4]. This minimal promoter encompasses the conserved junction sequence and is active in both defective interfering genomes and in SINV, although it is approximately threeto sixfold less active than the full active -98 to +14 promoter domain $[4,7]$. Two elements spanning positions -40 to -20 and +6 to +14 were found to be required for full promoter activity, and they act synergistically to enhance transcription [7]. Previous results suggest that $5^{\prime}$-proximal nucleotides relative to the $26 \mathrm{~S}$ mRNA start site are more important for the activity of the SP of SINV. The XJ-160 (GenBank accession No. AF103728) virus was used to investigate the relationship between the promoter sequence and its activity. XJ-160 is a Sindbis-like virus isolated from a pooled sample of Anopheles mosquitoes collected in Xinjiang, China, in 1990 [8]. Our results clearly demonstrate that the minimal SINV SP encompassing -19 to +5 of the start site is not always active, and that the $3^{\prime}$-proximal nucleotides relative to the $26 \mathrm{~S}$ mRNA start site are also important for the activity of the SP of SINV.

An alignment of SINV genomic sequences identified a 68-nucleotide conserved domain spanning positions -19 to +49 relative to the subgenomic mRNA start site. Similar to other members of the genus Alphavirus, 19 nucleotides upstream and two downstream of the initiation site are absolutely conserved among SINVs. However, a T nucleotide is found at -18 in all known SINVs, except for the XJ-160 virus, which has an A at -18 . In addition, the $\mathrm{XJ}-160$ virus has a $\mathrm{T}$ at +49 , while other SINVs have a $\mathrm{C}$ at +49 , which is positioned next to the initiation codon $(+50$ to +52$)$ of the viral structural proteins. To investigate the effects of the T nucleotide at -18 or +49 on the activity of the $26 \mathrm{~S}$ promoter and the function of the minimal SINV promoter, several SPs (SP1 to SP5) of the XJ-160 virus were designed as shown in figure 1. Promoter SP1 encompassing the domain -19 to +5 relative to the $26 \mathrm{~S}$ mRNA start site is identical to the SINV minimal 26S mRNA promoter. Compared with the wild-type promoter SP2 $(-19$ to +49$)$, SP3 and SP4 have an $\mathrm{A}$ to $\mathrm{T}$ change at -18 and $\mathrm{T}$ to $\mathrm{C}$ change at +49 , respectively. Promoter SP5 contains a deletion of the $\mathrm{T}$ at position +49 relative to SP2.

Recombinant plasmid pBR-XJ160 is an infectious fulllength cDNA clone of the XJ-160 virus, from which rescued virus can be obtained by transcription in vitro and transinfection [9]. Based on pBR-XJ160, recombinant clones with two $26 \mathrm{~S}$ promoters were constructed to investigate subgenomic mRNA promoter function: a wild-type $26 \mathrm{~S}$ promoter for expression of the structural protein, and a designed promoter, which was placed upstream of a reporter gene (enhanced green fluorescence protein (EGFP) 
or Gaussia luciferase (GLUC)). As can be seen in figure 1, the reporter gene cassettes consisted of a reporter gene (EGFP or GLUC), and the designed SPs were placed between the $3^{\prime}$-terminus of the virus structural genes and the $3^{\prime}$-untranslated region ( $3^{\prime}$ UTR). Recombinant clones carrying EGFP and SP1, SP2, SP3, SP 4 or SP 5 were designated pBR-XJ11, pBR-XJ12, pBR-XJ13, pBR-XJ14 or pBR-XJ15, respectively. Similarly, recombinant clones with GLUC and SP1, SP2, SP3, SP4 or SP5 were named pBR-XJ21, pBR-XJ22, pBR-XJ23, pBR-XJ24 or pBRXJ25, respectively. Rescued viruses could be obtained by transfecting $2 \mu \mathrm{g}$ RNA transcripts from the above recombinants into BHK-21 cells. The corresponding virus resulting from the transfection of clone pBR-XJ11 to pBRXJ15 was designated XJ-11 to XJ-15. Similarly, the rescued virus from pBR-XJ21 to pBR-XJ25 was named XJ-21 to XJ-25.

After the transfection of BHK-21 cells with the RNA transcripts from the recombinant clones, a typical cytopathic effect similar to that induced by XJ-160 virus could be seen $24 \mathrm{~h}$ postinfection (h p.i.), such as cell rounding and a loss of adherence (data not shown). However, no cytopathic phenotype was observed in BHK-21 cells infected with RNA transcripts from pBRXJ11 or pBR-XJ21 with an SINV minimal SP $(-19$ to +5$)$. To further examine the effects of the designed promoter on the propagation of XJ-160, growth curves of the recombinant viruses were constructed in BHK-21 cells. As shown in figure 2 , compared with XJ-160 virus, slightly higher titers of XJ-12 or XJ-13 virus were detected at $8-48$ h p.i. In contrast, lower titers of XJ-14 or XJ-15 virus were consistently detected during infection. The growth curve from XJ-21 to XJ-25 virus carrying GLUC and designed SPs had similar characteristics to that of the XJ-11 to XJ-15, respectively (data not shown). In addition, the growth tendencies of the mutant viruses were similar to that of XJ-160 virus. For evaluating the difference between the wild-type virus and mutant viruses, significant differences were performed using the oneway ANOVA analysis function in the SPSS software package (release 12.1; SPSS, Inc., Chicago, Ill., USA). Differences were considered significant at $\mathrm{p}<0.05$. These results indicate that the minimal SINV SP had no activity in XJ-160 virus, and that substitution of the T for A at -18 had no marked effect on the secretion of replicated virions, and slightly enhanced the propagation capacity of XJ-160 virus. In contrast, the differences between the mutant viruses with mutation at +40 (XJ-14 and $\mathrm{XJ}-15$ ) and XJ-160 virus were statistically significant at all indicated time points $(\mathrm{p}<0.05)$, suggesting that

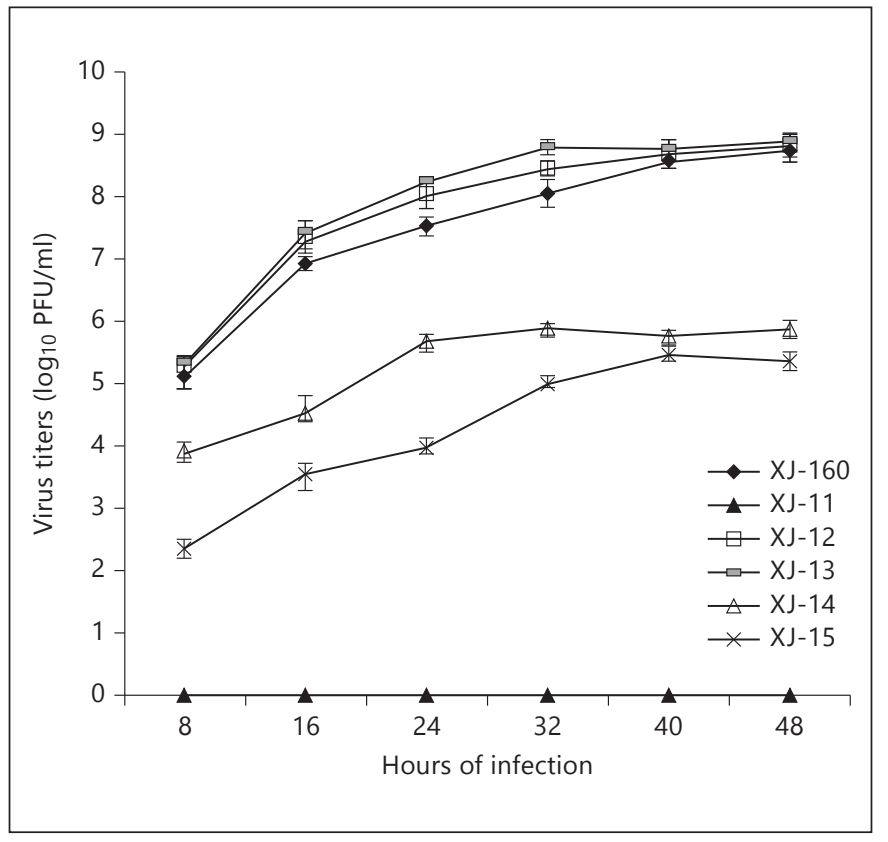

Fig. 2. Growth kinetics of mutant viruses in BHK-21 cells. BHK21 cells were infected with XJ-11 to XJ-15 or XJ-160 at a MOI of 0.01 . Samples of supernatant were removed at the indicated times and the virus yield evaluated by plaque assay. The data shown are from one of three representative experiments. Each point represents the mean titer of three independent wells with its standard deviation.

deletion or substitution of the $\mathrm{T}$ at +49 significantly decreased viral propagation capacity.

To further examine the relationship between the SP sequence and its function, reporter gene assays were performed as described previously [10]. Specific green fluorescence was observed in BHK-21 cells transfected with $\mathrm{XJ}-12$ to XJ-15 virus, while no EGFP expression was observed following transfection with pBR-XJ11 (fig. 3a), confirming the nonfunction of the SINV minimal SP in $\mathrm{XJ}-160$ virus. In BHK-21 cells infected with XJ-12 or XJ13 virus, continuously enhanced fluorescence could be seen 24-72 h p.i.; the EGFP expression peak was found at $48 \mathrm{~h}$ p.i. On the passaging of XJ-12 to XJ-15, EGFP expression was observed in cells infected with repeated passages of XJ-12 or XJ-13 virus, while no specific green fluorescence was observed after XJ-14 or XJ-15 virus had been passaged three times (data not shown). Similarly, continuously high luciferase activity was detected in BHK-21 cells infected with repeated passages of XJ-22 or $\mathrm{XJ}-23$ virus, while only background levels of luciferase were detected after XJ-24 or XJ-25 virus was passaged 

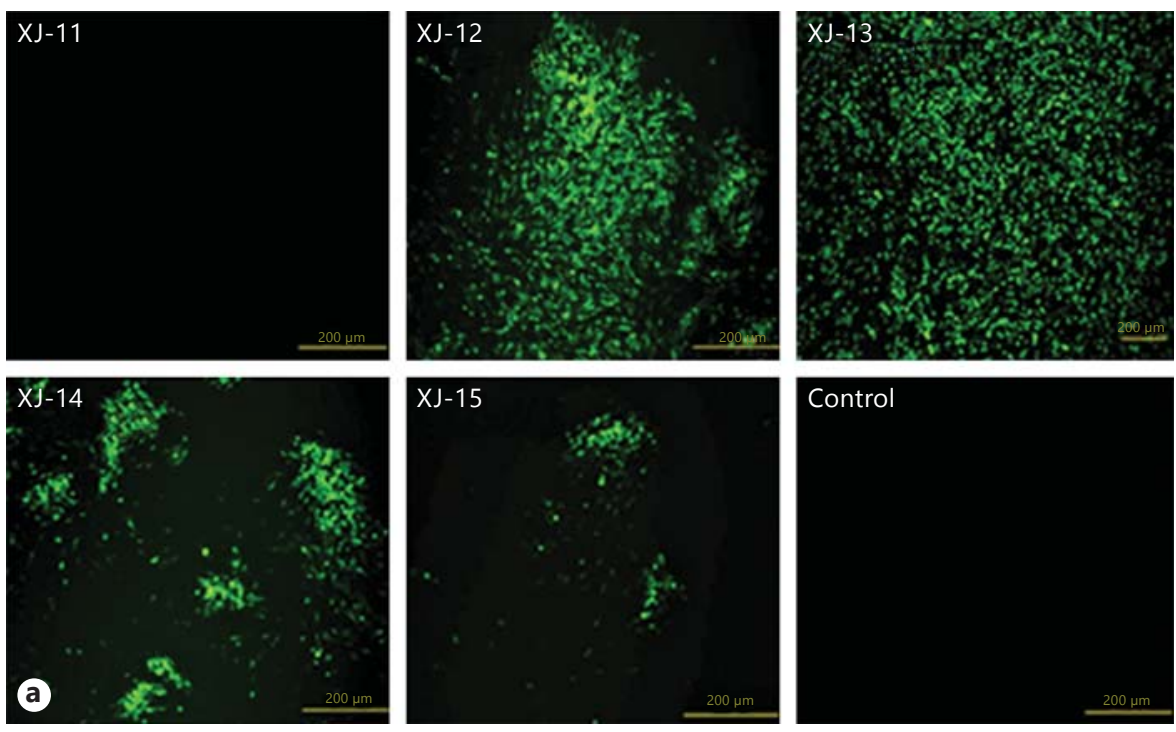

Fig. 3. Report gene assays. a Expression of EGFP in BHK-21 cells. BHK-21 cells were infected with $\mathrm{XJ}-12$ to $\mathrm{XJ}-15$ virus and RNA transcripts from $\mathrm{pBR}-\mathrm{XJ1}$, respectively, and EGFP expression observed using an inverted fluorescence microscope $(\times 400)$ at $44 \mathrm{~h}$ p.i. BHK-21 cells infected with XJ-160 virus were used as a control. b Expression of GLUC in BHK-21cells. BHK-21 cells were infected with repeated passages of XJ-21 to XJ-25 virus, and at the indicated passaging times, a GLUC assay was performed using a Gaussia Luciferase Assay System (Promega) at $24 \mathrm{~h}$ p.i. Each data point represents the mean \pm SEM of three independent experiments. RLU = Relative light units.

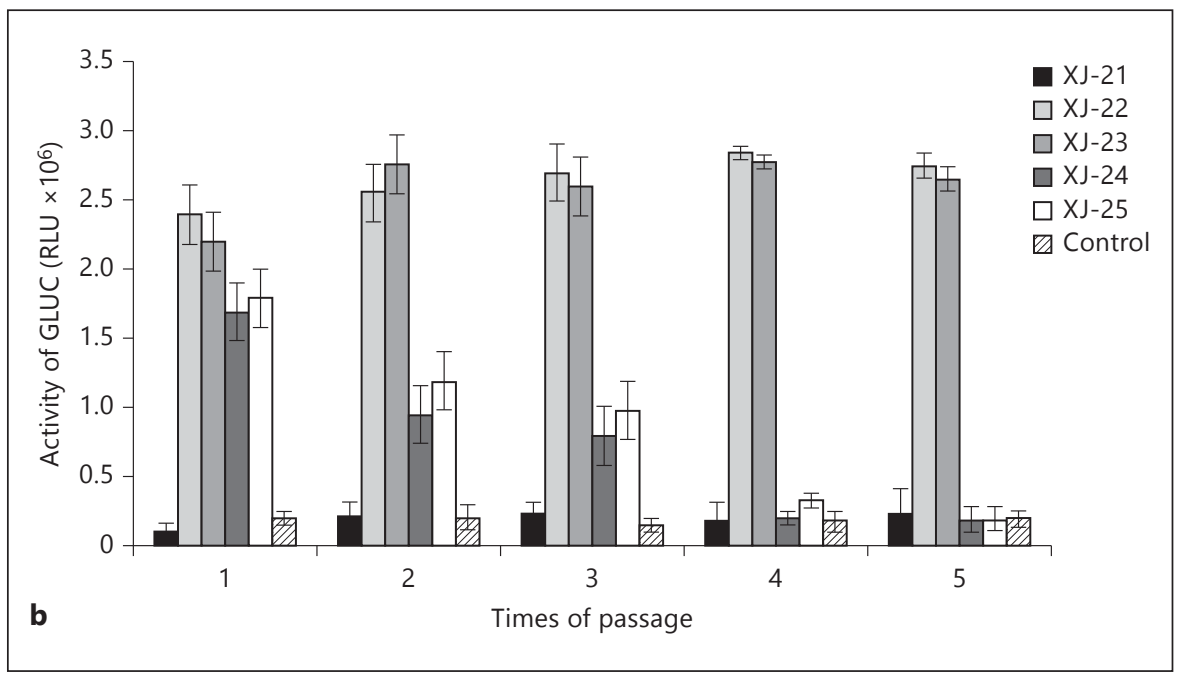

three times (fig. 3b). There were no significant differences among the activity numbers of GLUC expressed by the different passages of XJ-22 or XJ-23 virus ( $\mathrm{p}>0.05$ ). However, passage 1 of XJ-24 or XJ-25 virus exhibited significantly higher GLUC activity than the following passages of the corresponding virus $(\mathrm{p}<0.05)$. These results further demonstrate the distinct effects of the two T nucleotides in the $26 \mathrm{~S}$ mRNA promoter on the infectivity of XJ-160 virus.

For some positive-stranded RNA viruses, including picornaviruses and flaviviruses, genomic RNA is the only viral mRNA synthesized in infected cells. For many other positive-stranded RNA viruses, including alphaviruses and coronaviruses, one or more subgenomic RNAs are also synthesized in infected cells. Two distinct mech- anisms for generating subgenomic RNAs have been identified. First, subgenomic RNA synthesis by coronaviruses involves discontinuous RNA synthesis [11]. The second is the initiation of transcription at an internal site called an SP on the minus strand of the genomic RNA. The second mechanism is the most clearly established in alphaviruses $[6,12,13]$ and the plant virus brome mosaic virus (BMV) $[14,15]$. Thus, binding between RNAdependent RNA polymerase (RDRP) and SP may be a major contributor to the transcription of $26 \mathrm{~S}$ mRNA. nsP4, which has been identified as the Sindbis viral RDRP, is highly conserved in the genus Alphavirus, and plays a major role in viral RNA replication $[16,17]$. nsP4 also recognizes the SP, and the specific amino acid sequence in $\mathrm{nsP} 4$ responsible for recognizing $\mathrm{SP}$ is 


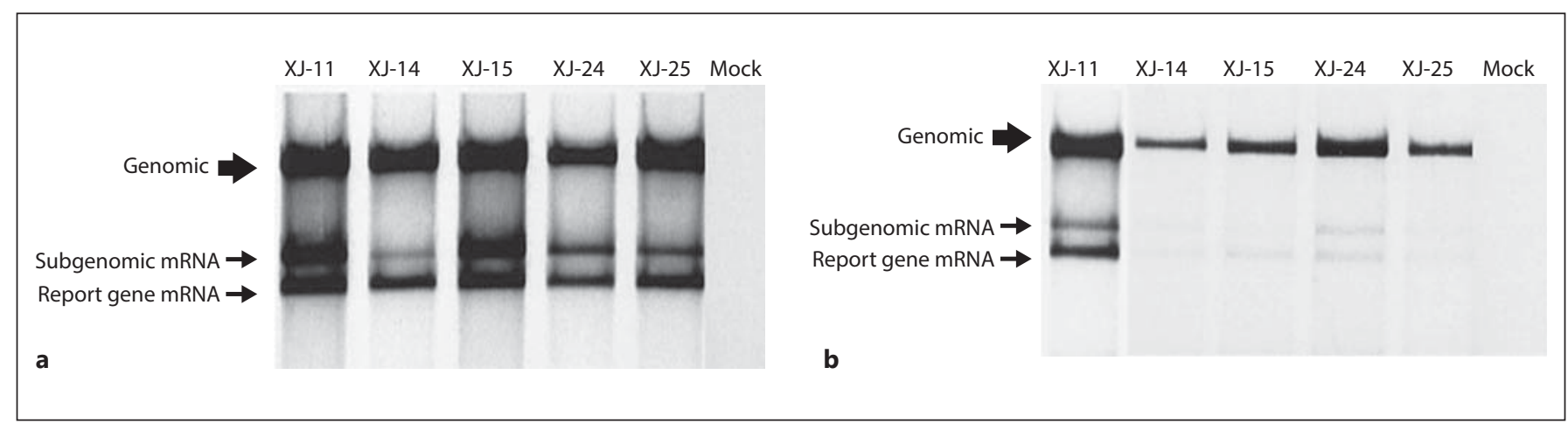

Fig. 4. Intracellular viral RNA analysis. BHK-21 cells were transfected with $2 \mu \mathrm{g}$ in vitro transcripts RNA. Dactinomycin was added $1 \mathrm{~h}$ before the cells were labeled with $\left[{ }^{3} \mathrm{H}\right]$ uridine (incorporation into viral RNAs) for $2 \mathrm{~h}$. Intracellular RNAs isolated at passages 1 and 3 from the cells and were denatured and separated through $1 \%$ agarose. The gel was treated for fluorography and exposed to film. The genomic RNA (big arrow) and the two subgenomic mRNAs (small arrows) were detected.
329-LVRRLT-334 [18]. Substitution of the T for A at -18 had little effect on the activity of the SP or the characteristics of XJ-160 virus. One explanation for this is that $\mathrm{RDRP}$ is able to tolerate sequence changes at this position.

Compared with substitution of the $\mathrm{T}$ for $\mathrm{A}$ at -18 , change or deletion of the $\mathrm{T}$ at +49 resulted in very low activity levels of report genes. Since the +49 mutation is positioned next to the initiation codon of the reporter genes, it is likely that the translatability of the second mRNA is altered by the change at the position of +49 . To confirm the contribution of +49 to promoter function, virus-specific RNAs synthesized by the recombinant virus both in passage 1 and 3 were analyzed as described previously by Hertz and Huang $[19,20]$. As shown in the figure 4, all the mutant RNA transcripts produce a genomic and two subgenomic mRNAs at passage 1. Compared with XJ-11 with the substitution at -18 , the levels of virus-specific RNAs from the RNA transcripts with the +49 mutation are obviously decreased at passage 3 , including XJ-14, XJ-15, XJ-24 and XJ-25. Especially, two subgenomic mRNAs are hardly detected in the BHK-21 cells infected with RNA transcripts with the +49 mutation, suggesting that the contribution of +49 mutations to promoter function may be related with the translatability of the second mRNA.

Host-dependent evolution of SINV promoter for subgenomic mRNA synthesis and evolution of SP in cultured cells were analyzed by Hertz and Huang $[19,20]$, which is the first report of construction of double SP cDNA clone of SINV. The analysis of subgenomic mRNA promoter function using a full-length cDNA clone of SINV is problematic. First, the clone is somewhat cumbersome to manipulate because of its large size and lack of unique restriction sites around the junction region. The second, and more serious, impediment is that the open reading frame of the nonstructural protein nsP4 overlaps with the initiation site for subgenomic RNA synthesis. Full-length pBR-XJ160 is 14,625 bp, and no reasonable unique restriction sites for the construction of a recombinant clone with two promoters have been found. Two unique restriction sites, a KasI site at the $3^{\prime}$-terminal end of the E1 gene and a XhoI site at the $3^{\prime}$ terminal of the polyA tail, were used to construct the desired clones in this study. These were generated by three long-fragment fusion PCRs and two ligation reactions. In addition, the designed SPs were placed upstream of the reporter genes, which avoided the effects of nsP4 overlap on the subgenomic mRNA promoter. In this way, we constructed competent viruses expressing EGFP or GLUC, such as XJ-12, XJ-13, XJ-22 and XJ-23. These represent a powerful tool for studying the replication strategy and pathogenetic mechanisms of alphaviruses.

\section{Acknowledgements}

We thank Dr. Ting Zhao for the experiment of intracellular viral RNA analysis. This work was supported by grants from the National Natural Science Foundation of China (No. 30970 160; 81160353), China Mega-Project for Infectious Disease (2011ZX10004-001), and the Development Grant of State Key Laboratory for Infectious Disease Prevention and Control (2011SKLID205). 


\section{References}

1 Vogel P, Fritz DL, Kuehl K, Davis KJ, Geisbert T: The agents of biological warfare. JAMA 1997;278:438-439.

- 2 Burgess TH, Steele KE, Schoneboom BA, Grieder FB: Clinicopathologic features of viral agents of potential use by bioterrorists. Clin Lab Med 2001;121:475-493.

-3 Strauss JH, Strauss EG: The alphaviruses: gene expression, replication, and evolution. Microbiol Rev 1994;58:491-562.

4 Levis R, Schlesinger S, Huang HV: Promoter for Sindbis virus RNA-dependent subgenomic RNA transcription. J Virol 1990;64:1726-1733.

5 Hertz JM, Huang HV: Utilization of heterologous alphavirus junction sequences as promoters by Sindbis virus. J Virol 1992;66:857-864.

-6 Ou J-H, Rice CM, Dalgarno L, Strauss EG, Strauss JH: Sequence studies of several alphavirus genomic RNAs in the region containing the start of the subgenomic RNA. Proc Natl Acad Sci USA 1982;79:5235-5239.

7 Raju R, Huang HV: Analysis of Sindbis virus promoter recognition in vivo, using novel vectors with two subgenomic mRNA promoters. J Virol 1991;65:2501-2510.
8 Liang GD, Li L, Zhou GL, Fu SH, Li QP, Li FS, $\mathrm{He} \mathrm{HH}$, Jin Q, He Y, Chen BQ, Hou YD: Isolation and complete nucleotide sequence of a Chinese Sindbis-like virus. J Gen Virol 2000; 81:1347-1351.

9 Yang YL, Liang GD, Fu SH, He H, Li X, Deng J, Su N, Wang L, Hou YD: Construction and infection analysis of the full-length cDNA clone of XJ-160 virus, the first Sindbis virus isolated in China. Virol Sin 2005;21:173-180.

10 Zhu WY, Li JJ, Tang L, Wang HQ, Li J, Fu JJ, Liang GD: Glycoprotein is enough for Sindbis virus-derived DNA vector to express heterogenous genes. Virol J 2011;8:344.

11 Baric RS, Stohlman SA, Razavi MK, Lai MMC: Characterization of leader-related small RNAs in coronavirus-infected cells: further evidence for leader-primed mechanism of transcription. Virus Res 1985;3:19-33.

12 Rice CM, Strauss JH: Nucleotide sequence of the 26S mRNA of Sindbis virus and deduced sequence of the encoded virus structural proteins. Proc Natl Acad Sci USA 1981;78:20622066.

13 Strauss EG, Rice CM, Strauss JH: Complete nucleotide sequence of the genomic RNA of Sindbis virus. Virology 1984;133:92-110.

14 French R, Ahlquist P: Characterization and engineering of sequences controlling in vivo synthesis of brome mosaic virus subgenomic RNA. J Virol 1988;62:2411-2420.
15 Marsh LE, Dreher TW, Hall TC: Mutational analysis of the core and modulator sequences of the BMV RNA3 subgenomic promoter. Nucleic Acids Res 1988;16:981-995.

16 Thal MA, Wasik BR, Posto J, Hardy RW: Template requirements for recognition and copying by Sindbis virus RNA-dependent RNA polymerase. Virology 2007;358:221-232.

17 Rubach JK, Wasik BR, Rupp JC, Kuhn RJ, Hardy RW, Smith JL: Characterization of purified Sindbis virus nsP4 RNA-dependent RNA polymerase activity in vitro. Virology 2009; 384:201-208.

18 Li ML, Stollar V: Identification of the amino acid sequence in Sindbis virus nsP4 that binds to the promoter for the synthesis of the subgenomic RNA. Proc Natl Acad Sci USA 2004; 101:9429-9434.

19 Hertz JM, Huang HV: Evolution of the Sindbis virus subgenomic mRNA promoter in cultured cells. J Virol 1995;69:7768-7774.

20 Hertz JM, Huang HV: Host-dependent evolution of the Sindbis virus promoter for subgenomic mRNA synthesis. J Virol 1995;69:77757781. 